\title{
AVO analysis using horizontal component of seismic data in equivalent offset migration method
}

\author{
Tomoaki TANAKA ${ }^{1}$, Hitoshi MIKADA ${ }^{1}$ and Junichi TAKEKAWA ${ }^{1}$ \\ ${ }^{1}$ Dept. of Civil and Earth Res. Eng., Kyoto University
}

\begin{abstract}
For exploring subsurface resources such as oil or natural gas reservoirs, seismic reflection survey has been widely implemented in order to image subsurface structures. In recent years, utilization of S-wave or converted wave is required for estimating lithology or petrophysical properties of reservoir rock. However, such an analysis of S-wave seismograms had been relatively difficult. On the other hand, equivalent offset migration (EOM) is one of the prestack time migrations and has been found to be effective method for imaging S-wave information on the common scatter point (CSP) gather with recorded horizontal component in our previous study. Furthermore, S-wave AVO effect has also been confirmed by the amplitude reversal of S-wave event on the CSP gathers. Therefore, we propose the procedure of accurate estimation of densities and shear modulus with S-wave source. First, we conduct numerical experiment with a 2D layer model using horizontal point force to obtain horizontal component seismic data, in which we can get higher S/N data about S-wave. Second, we implement EOM with those data to get CSP gather, and calculate each cross-correlated value versus incident angle as observed waveform information. Third, in contrast, we generate calculated waveform information as a function of incident angle and physical properties with geometrical spreading, radiation pattern and S-wave reflection coefficient. Finally, we can estimate the optimal solutions by minimizing the misfit from the both information.
\end{abstract}

\section{INTRODUCTION}

Seismic migration is one of the most efficient and major techniques in seismic reflection survey for exploring hydrocarbon reservoirs. In recent years, prestack time migration ${ }^{1,2)}$ is used as a tool for high-accuracy imaging of complicated subsurface structure, and an equivalent offset migration (EOM) technique, one of prestack time migration, has been proposed as a method equivalent to partial prestack migration with much simpler and much lower-cost computational algorithm ${ }^{3)}$.

It is important for estimating lithological properties to obtain $\mathrm{S}$-wave velocity structures, but there have not been well estimating techniques in the current practice of seismic imaging. We found out that EOM with horizontal component waveform could be used to extract S-wave information efficiently for the estimation of S-wave velocities. We noticed the amplitude reversal due to $\mathrm{S}$-wave AVO effects in a common scatter point (CSP) gather of EOM using horizontal component ${ }^{4}$. On this point, the application of EOM would be useful not only for estimating accurate velocities but for the AVO analysis for estimating S-wave properties. We conduct numerical experiment in a layer model using horizontal point force to get horizontal component records and the result shows possibility of estimating physical properties of the layer under the geological boundary with AVO analysis using $\mathrm{S}$-wave CSP gather.

\section{METHOD}

First, we conduct numerical simulation of seismic reflection survey in order to obtain received records of horizontal component as observed information. After that, we apply the EOM technique to those records with making the CSP gather to extract $\mathrm{S}$-wave AVO effect information. Since the S-wave AVO effect includes reflected wave information from each geological boundary, we try to estimate the information about $\mathrm{S}$-wave velocity structure from the gather. In typical CSP gathers, S-wave reflection event has an amplitude reversal point due to the AVO effect. The amplitude formation could include information about subsurface boundaries. Therefore, we take cross-correlation of CSP gather traces versus incident angle to indicate the change of amplitude as a function of incident angle. The estimated subsurface properties are derived from an analytical solution by fitting to the observed one.

Equation $1^{3)}$ is used for making CSP gather. 


$$
h_{e}^{2}=x^{2}+h^{2}-\left(\frac{2 x h}{t V_{m i g}}\right)^{2}
$$

In equation $1, h_{e}$ is equivalent offset, $h$ is half offset between shot point and receiver point, $x$ is horizontal distance between scattering point and the midpoint of shot point and receiver point, $t$ is two-way travel time and $V_{\text {mig }}$ is migration velocity.

Figure 1 is an example of CSP gathers as a result of EOM using horizontal component when the common scatter point is on the middle of the model. $\mathrm{S}$-wave AVO effect is confirmed from the flipped point of amplitude on around $h_{e}=750 \mathrm{~m}$ and $t=$ $1.8 \mathrm{sec}$.

The analytical solution is calculated by taking account of the geosphirical divergence, the S-wave source radiation pattern, and $\mathrm{S}$-wave reflection coefficient $^{5)}$ with the incident angle.

We invert exact physical properties in the subsurface to minimize a misfit function as shown below.

$$
\begin{aligned}
& M=\int_{0}^{\theta_{\mathrm{L}}}\left(\mathrm{C}_{\mathrm{obs}}(\theta)\right. \\
& \left.-\mathrm{C}_{\mathrm{cal}}\left(\theta ; \rho_{1}, \rho_{2}, V_{S 1}, V_{S 2}, V_{P 1}, V_{P 2}\right)\right)^{2} \mathrm{~d} \theta
\end{aligned}
$$

$\mathrm{C}_{\text {obs }}$ and $\mathrm{C}_{\text {cal }}$ are cross-correlated values as functions of incident angles using observed and synthetic data sets, respectively, $\theta_{L}$ is the maximum incident angle. A set of optimal properties is numerically led by using the conjugate gradient method.

\section{NUMERICAL MODEL AND RESULTS}

Fist, we simulate 2D elastic wave propagation with finite difference method using fourth-order staggered grid $^{6}$. The numerical model we used is shown in Figure 2. The upper boundary is set as the free boundary, and the others are set C-PML absorbing boundary condition ${ }^{7}$. The survey line is on the point from $500 \mathrm{~m}$ apart from the edge. 201 sources and receivers are set at the surface with a constant interval of $20 \mathrm{~m}$. We use the second derivative Gaussian wavelet as horizontal source and record horizontal component data on each receiver.

We calculate the misfit of cross-correlated values of those observed and calculated data using equation 2 . The misfit of the minimization is shown

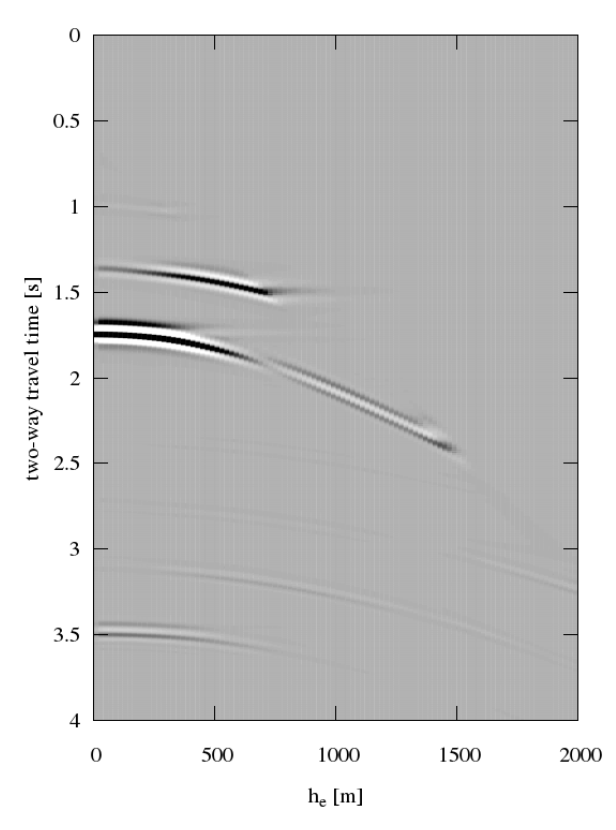

Figure 1 This is an example of CSP gather. It shows the scattered energy from scattering points on the same vertical line. The third event from the top is $\mathrm{S}$-wave scattered event.

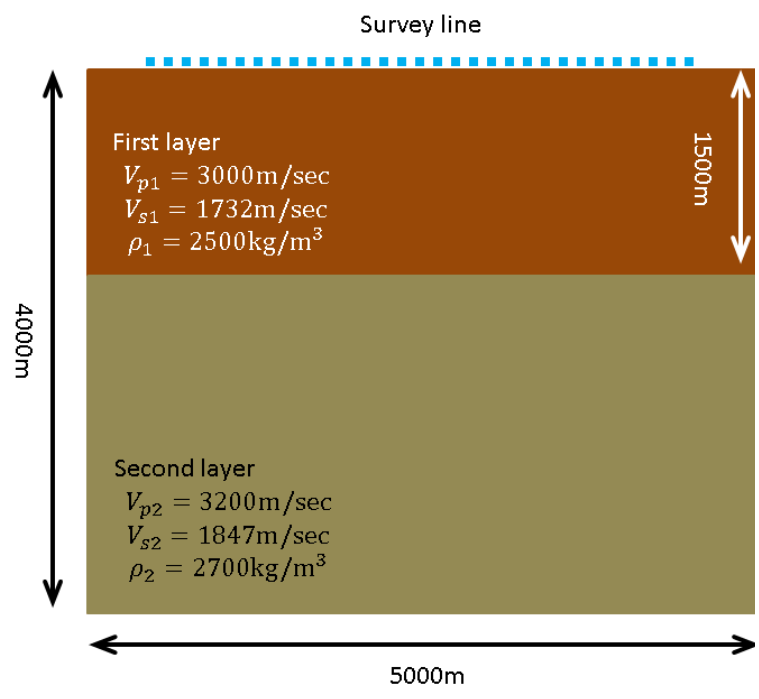

Figure 2 Numerical model for seismic reflection survey simulation

in Figure 3, and the result of minimization is shown in Figure 4. Initial values of density and shear modulus are set to about $10 \%$ lower than the exact solution. The inverted solutions converge to the exact values after 8 iterations. 


\section{CONCLUSION}

In our present study, we proposed a progressive approach to estimate $\mathrm{S}$-wave velocity structure using CSP gather by utilizing the AVO effect. Comparing with the original EOM, our method is defined by the use of the horizontal source and the horizontal component of received data. Numerical results show the possibility of simultaneous estimation of density and shear modulus efficiently. This result indicates that the lithological properties of the subsurface materials could be estimated by the horizontal records in the surface reflection survey.

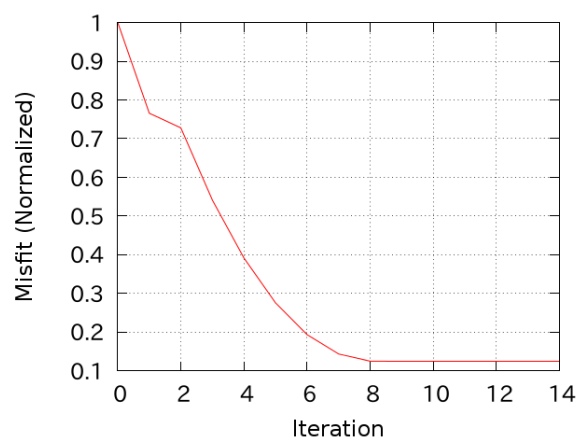

Figure 3 This shows the misfit of minimization. The misfit is normalized by the initial one.
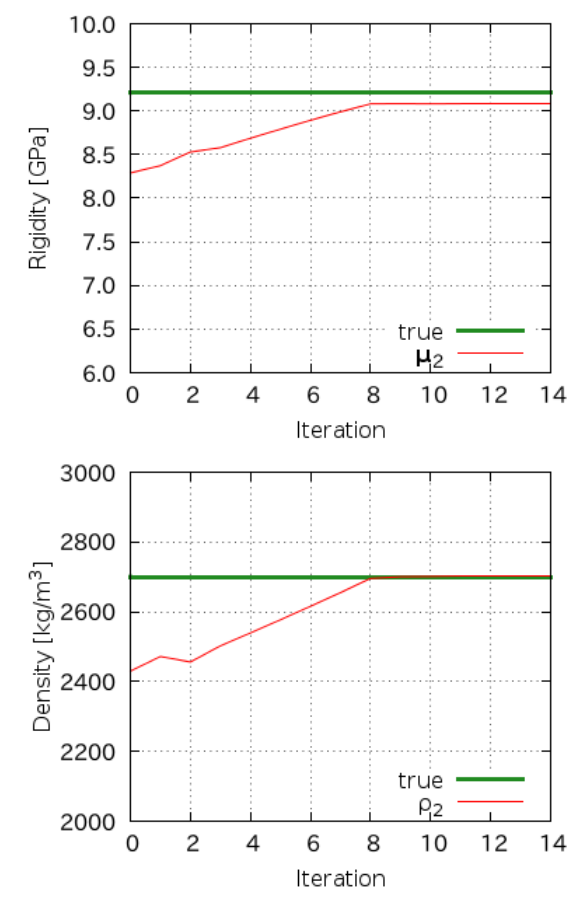

Figure 4 This is the result of estimation.

\section{REFERENCES}

1) Hale, D., 1984, Dip moveout by Fourier transform, Geophysics, 49, 741-757.

2) Gardner, G. F. H., Wang, S. Y., Pan, N. D., and Zhang, Z., 1986, Dip moveout and prestack imaging, 18th Ann. Offshore Tech. Conf., Expanded Abstracts, 71-75.

3) Bancroft, J. C., Geiger, H. D., and Margrave, G. F., 1998, The equivalent offset method of prestack time migration, Geophysics, 63, 2042-2053.

4) Tanaka, T., Mikada, H., Takekawa, J., Elastic imaging of subsurface Structure with Equivalent Offset Migration for multicomponent seismic data, Recent Advances in Exploration Geophysics 2016, 2016. doi:10.3997/2352-8265.20140205

5) Aki, K. and Richards, P. G., 2002, QUANTITATIVE SEISMOLOGY SECOND EDITION, University Science Books, 179-185

6) Virieux, J., 1986, P-SV wave propagation in heterogeneous media, Velocity-stress finite-difference method, Geophysics, 51, 889-901

7) Komatitsch, D., and Martin, R., 2007, An unsplit convolutional perfectly matched layer improved at grazing incidence for the seismic wave equation, Geophysics, 72, 155-167 